\title{
Efeito da inoculação de biorremdiador no procedimento de compostagem de lodo de esgoto
}

O Brasil infelizmente ainda enfrenta problemas gravíssimos na atualidade relacionados ao gerenciamento de resíduos sólidos em vários setores. Entre os mais diversos resíduos produzidos diariamente, o lodo de esgoto sanitário, merece destaque especial devido à sua disposição final problemática e frequentemente negligenciada. A grande preocupação resultante do mau gerenciamento deste tipo de resíduo, está no fato de ser altamente contaminante, por apresentar elevada quantidade de microrganismos patogênicos e metais tóxicos, prejudiciais à saúde humana e do meio ambiente. No entanto, como alternativa, comumente entre os tratamentos e disposições finais, o que mais se evidencia é a redução de volume e umidade para disposição em aterro sanitário. Entretanto, cabe ressaltar, que atualmente, não há como negar algumas prerrogativas que vão de encontro a este tipo de possibilidade. Além da escassez de áreas aptas à construção e aterros sanitários, devemos considerar as imposições da Lei № $12.305 / 10$, regulamentada pelo decreto № $7.404 / 10$, de 23 de dezembro de 2010 , que após sua promulgação, proibiu a disposição final de resíduos sólidos em aterros sanitários. Desse modo, a bioxidação de lodos de esgoto sanitário por meio do processo de compostagem, torna-se uma técnica vantajosa, uma vez que proporciona a melhoria das qualidades físicas, químicas e biológicas do resíduo, além de representar uma alternativa promissora em relação aos fertilizantes químicos. Neste sentido, este estudo apresenta resultados do processo de compostagem de lodo de esgoto sanitário por meio da inoculação de biorremediador (acelerador de compostagem). 0 objetivo deste estudo foi avaliar a eficiência do biorremediador no processo de compostagem quando comparado ao processo sem inoculação. Para isso, durante o processo de compostagem foram realizadas analises de temperatura, umidade, $\mathrm{pH}$, condutividade elétrica e teores de sólidos (totais, fixos e voláteis). Ante o exposto, os resultados das análises de temperatura, umidade, $\mathrm{pH}$ condutividade elétrica e sólidos, efetuadas durante o processo de compostagem, não demostraram melhoras significativas que justifiquem o uso do biorremediador quando confronta (a) a inoculação do biorremediador não proporcionou melhoras significativas no sistema de compostagem a ponto de justificar seu uso, uma vez que os microrganismos nativos presentes no sistema são suficientes para o processo de degradação da matéria orgânica.

\section{Effect of inoculation of biorremediador in the procedure of composting sewage sludge}

\begin{abstract}
Brazil unfortunately still faces very serious problems currently related to the management of solid waste in various sectors. Among the most diverse waste produced daily, sewage sludge deserves special mention due to its problematic and often neglected final disposition. The great concern resulting from the poor management of this type of waste is the fact that it is highly contaminating due to the high amount of pathogenic microorganisms and toxic metals that are harmful to human health and the environment. However, as an alternative, commonly between treatments and final provisions, what is more evident is the reduction of volume and humidity for disposal in landfills. However, it should be emphasized that, currently, there is no denying certain prerogatives that go against this type of possibility. In addition to the shortage of suitable areas for construction and landfills, we must consider the provisions of Law No. 12,305 / 10, regulated by Decree No. 7.404 / 10, of December 23, 2010, which, after its promulgation, prohibited the final disposal of solid wastes in landfills. Thus, the biooxidation of sanitary sewage sludge through the composting process becomes an advantageous technique, since it improves the physical, chemical and biological qualities of the waste, besides representing a promising alternative in relation to fertilizers chemicals. In this sense, this study presents results of the composting process of sanitary sewage sludge by means of the inoculation of a bioremediator alternative in relation to fertilizers chemicals. In this sense, this study presents results of the composting process of sanitary sewage sludge by means of the inoculation of a bioremediator
(compost accelerator). The objective of this study was to evaluate the efficiency of the bioremediator in the composting process when compared to the process without inoculation. For this, during the composting process, analyzes of temperature, humidity, $\mathrm{pH}$, electrical conductivity and solids contents (total, fixed and volatile) were performed. The results of the analysis of temperature, humidity, $\mathrm{pH}$, electrical conductivity and solids, carried out during the composting process, did not show significant improvements that justify the use of the bioremediato when compared to the results obtained in the process without inoculation. Thus, in view of the conditions in which the study was developed, considering the type of sludge and structural material used, the inoculation of the bioremediator did not provide significant improvements in the composting system to the point of justifying its use, since the native microorganisms present in the system are sufficient for the degradation process of organic matter.
\end{abstract}

Keywords: Biorremediador; Composting; Inoculant; Sewage Slugde.

Topic: Engenharia Sanitária

Reviewed anonymously in the process of blind peer
Received: $10 / 06 / 2018$

Approved: $24 / 07 / 2018$
Welliton Leandro de Oliveira Boina (id

Universidade Federal de São Carlos, Brasil http://lattes.cnpq.br/8710752577794108 http://orcid.org/0000-0001-6896-5157

weboina@gmail.com

João Sérgio Cordeiro (iD

Universidade Federal de São Carlos, Brasil http://lattes.cnpq.br/2572864522562875 http://orcid.org/0000-0002-6294-8871

cordeiro.js@gmail.com

Rosane Freire Boina

Universidade Estadual Paulista, Brasil

http://lattes.cnpq.br/0004928784504087

http://orcid.org/0000-0002-9903-5902

rofreire@gmail.com

\section{Referencing this:}

BOINA, W. L. O.; CORDEIRO, J. S.; BOINA, R. F.. Efeito da inoculação de biorremdiador no procedimento de compostagem de lodo de esgoto. Revista Ibero Americana de Ciências Ambientais, v.9, n.5, p.106-116, 2018. DOI: http://doi.org/10.6008/CBPC2179-6858.2018.005.0011 


\section{INTRODUÇÃO}

A compostagem, segundo Fernandes et al. (1999), Paz et al. (2003), Budzialk et al. (2004), Insam et al. (2007) e Qian et al. (2014), pode ser definida como um processo de bioxidação aeróbica exotérmica de um substrato orgânico heterogêneo, no estado sólido em que os compostos orgânicos sofreram mineralização e processos de neo-síntese, assumindo natureza coloidal, denominado composto.

Neste sentido, a matéria orgânica é utilizada por microrganismos termófilos ou mesófilos aeróbios, resultando na produção de $\mathrm{CO}_{2}, \mathrm{H}_{2} \mathrm{O}$, substâncias minerais e matéria orgânica estável. A higienização ou eliminação dos microrganismos patogênicos presentes no lodo está diretamente ligada à elevação de temperatura durante a oxidação e o tempo de exposição.

Assim, de acordo com Carvalho (2002) e Fels et al. (2014), como resultado deste processo de bioxidação, observa-se a produção de um composto de fácil manipulação, isento de microrganismos patogênicos, estabilizado e higienizado, de coloração escura e odor não agressivo e aspecto agradável, rico em matéria orgânica umidificada com grande aptidão agronômica, fornecendo nutrientes às plantas e atuando como condicionador de solos.

Considerando o exposto, os biorremediadores, também referidos como inoculantes, são produtos biotecnológicos, geralmente na forma líquida, pelo qual sistemas biológicos são utilizados para tratar a poluição e restaurar a qualidade ambiental por meio da degradação dos poluentes. Possui como base a biorremediação, que de acordo com Andrade et al. (2010), compreende o uso de microrganismos externos, via inoculação, que podem ser adicionados ao processo de compostagem, geralmente compreendidos por fungos e bactérias, de ocorrência natural, nativos ou cultivados, para decompor ou reter contaminantes em resíduos, com a finalidade favorecer e incrementar a velocidade natural de degradação, resultando na compostagem acelerada.

Entretanto, constata-se na literatura, inúmeros estudos e pesquisas relativos à utilização de biorremediador na compostagem. Esta utilização, com a intenção de acelerar o processo natural ou para obter um produto final de melhor qualidade, tem sido um assunto um tanto controverso, logo, motivo deste estudo.

\section{MATERIAIS E MÉTODOS}

O estudo foi desenvolvido no pátio de compostagem da Estação de Tratamento de Esgoto Lageado (ETE-Lageado) da Companhia de Saneamento Básico do Estado de São Paulo (Sabesp), onde foi instalada uma estufa de compostagem (Figura 1) com as seguintes dimensões: 12,8 m (largura) x 81,0 m (comprimento) $x$ 6,5 m (altura), correspondendo a uma área de 1.036,8 $\mathrm{m}^{2}$. A ETE-Lageado está localizada em uma área cedida de 14 ha, dentro da Fazenda Experimental Legeado, sede da Faculdade de Ciências Agronômicas (FCA), da Universidade Estadual Paulista (Unesp), em Botucatu, SP. 




Figura 1: Vista aérea da Estufa de compostagem e ETE Lageado. Fonte: Google Earth (2018).

Atualmente, conforme informações obtidas no escritório da Sabesp localizado na ETE-Lageado, a unidade de tratamento produz em média cerca de $12 \mathrm{~m}$ / dia de lodo de esgoto úmido ( $80 \%$ de umidade), gerado nos Reatores UASB e que são encaminhado para centrifuga após receber polieletrólito, deste modo, uma geração em torno de $360 \mathrm{~m}^{3} / \mathrm{mês}$.

Para a montagem da leira de compostagem, foram utilizados $40 \mathrm{~m}^{3}$ de lodo de esgoto sanitário (Figura 2), obtido do sistema de tratamento da própria ETE-Lageado. Como fonte de carbono (material estruturante), foram utilizados $40 \mathrm{~m}^{3}$ de casca de eucalipto (Figura 3), obtido na Empresa Duratex S.A., localizada em Botucatu, SP.



Figura 2: Lodo de esgoto centrifugado após receber polieletrólito.

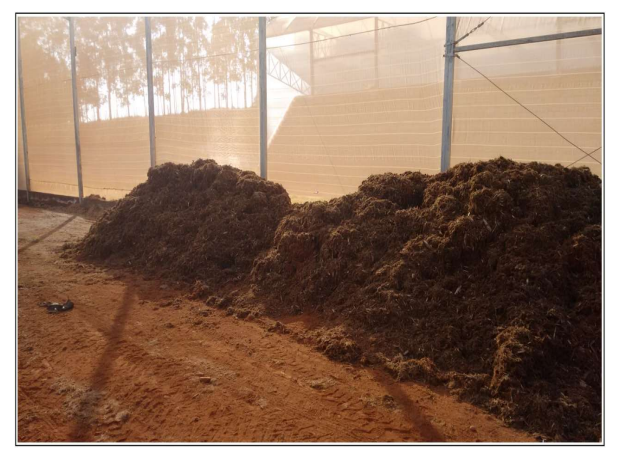

Figura 3: Casca de eucalipto usado como material estruturante e fonte de carbono.

Em relação a proporção carbono/nitrogênio $(C / N)$, foi utilizada uma relação de 1:1, considerando recomendações, estudos (custos e receitas) e experiências anteriores desenvolvidas pelo grupo de pesquisa do Projeto de Compostagem (Sabesp/Fapesp/Unesp) sob Coordenação do Prof. Dr. Roberto Lyra Villas-Bôas da FCA/Unesp de Botucatu, SP. A Figura 4, apresenta a mistura em relação C/N de 1:1.

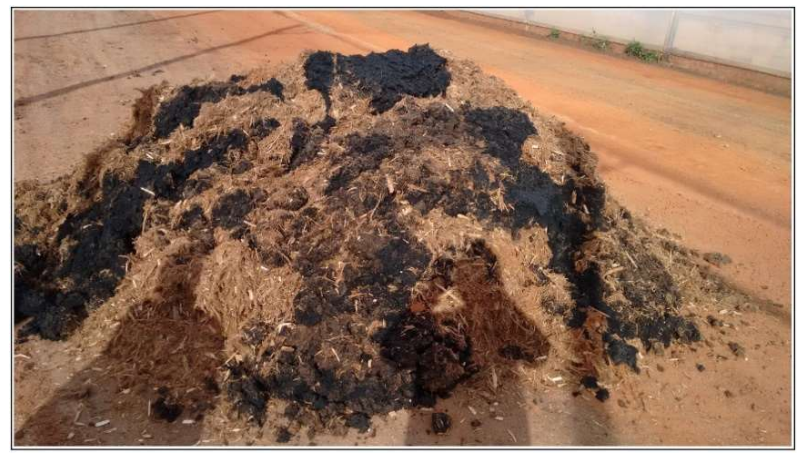

Figura 4: Relação C/N 1:1 - lodo de esgoto e casca de eucalipto. 
Após toda casca de eucalipto $\left(40 \mathrm{~m}^{3}\right)$ ser intercalada com todo o lodo de esgoto $\left(40 \mathrm{~m}^{3}\right)$, e esse material ser depositado sequencialmente dentro da estufa de compostagem, obteve-se a formação de uma leira de compostagem de $26 \mathrm{~m}$ (comprimento) x $3 \mathrm{~m}$ (largura) x $2 \mathrm{~m}$ (altura), correspondendo a um volume total de $80 \mathrm{~m}^{3}$. A Figura 5 apresenta a leira de compostagem.

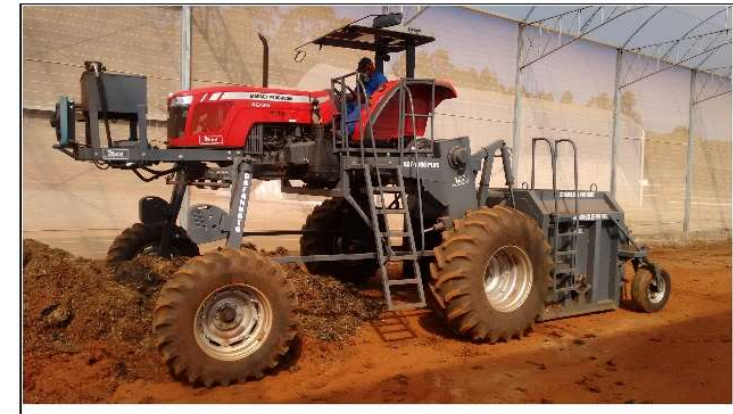

(a)



(b)

Figura 5: Formação da leira de compostagem.

Para inoculação do biorremediador comercial 'Acelerador de compostagem' da Embiotic ${ }^{\circledR}$-Line, a leira de compostagem, correspondente a $26 \mathrm{~m}$ de comprimento, foi dividida em duas partes iguais de $13 \mathrm{~m}$ (comprimento) $\times 3 \mathrm{~m}$ (largura) $\times 2 \mathrm{~m}$ (altura) cada uma. Dentre estas duas partes, uma parte foi utilizada para inoculação do biorremediador e a outra como controle, sem inoculação, para fins de comparação, durante e ao fim do processo de compostagem. Partindo do princípio que a relação $\mathrm{C} / \mathrm{N}$ adotada foi a de 1:1, utilizouse em cada parte da leira (13 m), a proporção de $20 \mathrm{~m}^{3}$ de lodo de esgoto juntamente com $20 \mathrm{~m}^{3}$ de casca de eucalipto, correspondendo no total a $40 \mathrm{~m}^{3}$ de material a ser compostado em cada parte da leira.

Antes de cada revolvimento da leira de compostagem, efetuada de forma mecanizada, eram inoculados $40 \mathrm{~L}$ de solução ativa de biorremediador, por meio de um regador. A rega foi feita uniformemente por toda a leira, desde a base até o topo da pilha de compostagem. Neste sentido, cada metro cúbico da massa em compostagem recebeu 1 litro de solução ativa, o que é suficiente para o processo, conforme informações obtidas pelo canal de atendimento 'fale conosco' da Korin Meio Ambiente (KMA).

Após a rega, tanto a parte inoculada quanto a parte controle eram revolvidas. Entretanto os revolvimentos foram feitos, sempre que a leira de compostagem apresenta-se temperatura (acima de $45^{\circ} \mathrm{C}$ ) e umidade (acima de 50\%), mas sempre as duas partes da leira (inoculada e controle) eram revolvidas no mesmo dia e horário. Em relação as análises, compreenderam a obtenção do teor de umidade, teor de sólidos totais (ST), teor de sólidos voláteis (SV) e teor de sólidos fixos (SF), potencial hidrogeniônico (pH) e condutividade elétrica (C.E.). A seguir, será apresentado os procedimentos adotados para cada análise.

\section{Série Sólidos}

Para a determinação da série sólidos (totais, fixos e voláteis), para cada método de determinação, foi utilizado a Norma NBR 10644/1989, Águas - Determinação de Resíduos (Sólidos) - Método Gravimétrico. 


\section{Sólidos Totais}

A determinação de Sólidos Totais (ST), foi obtida por meio do Método J - Resíduo total (sólidos totais) - Modificados para lodos e sedimentos conforme a NBR 10644/1989. Assim, primeiramente preparou-se as cápsulas de porcelanas. As capsulas foram colocadas em forno mufla a $(550 \pm 50)$ ํC por 1 h. Após esse tempo, as capsulas foram esfriadas no dessecador até alcançarem a temperatura ambiente. Logo após cada capsula foi pesada com precisão aproximada de $10 \mathrm{mg}$ e deixada no dessecador até o momento do uso.

Cada amostra foi transferida respectivamente para cada capsula (o peso das amostras variou entre 25 a $50 \mathrm{~g}$ ), e pesadas com precisão de $10 \mathrm{mg}$. Como as amostras se enquadram como amostra sólida, conforme a NBR 10664/1989, as amostras foram secas em estufa a 103ㅇ C por 12h, onde após esse tempo foram esfriadas em dessecador até a temperatura ambiente e pesadas com precisão de $10 \mathrm{mg}$. 0 teor de sólidos totais, foi obtido pela seguinte expressão:

$$
\% \text { resíduo total }=\frac{m 2 \times 100}{m 1}
$$

Onde:

$\mathrm{m} 2$ = massa do resíduo total, em $\mathrm{g}$; $\mathrm{m} 1$ = massa da amostra, em $\mathrm{g}$.

Consequentemente, por meio do resultado (\% resíduo total), conforme o Método J, foi possível determinar o teor de umidade, a qual foi obtida pela seguinte expressão:

Teor de umidada $=1-(\%$ teor de sólidos $)$

\section{Sólidos Fixos}

A determinação de Sólidos Fixos (SF), foi obtida por meio do Método L - Resíduo fixo (sólidos fixos) - Modificados para lodos e sedimentos conforme a NBR 10644/1989. Os resíduos, obtidos conforme o


temperatura ambiente em dessecador e pesados com precisão de $10 \mathrm{mg}$. O teor de sólidos fixos, foi obtido pela seguinte expressão:

$$
\% \text { resíduo fixo }=\frac{m 3 \times 100}{m 2}
$$

\section{Sólidos voláteis}

A determinação de Sólidos Voláteis (SV), foi obtida por meio do Método M - Resíduo volátil (sólidos voláteis) - Modificados para lodos e sedimentos conforme a NBR 10644/1989. O teor de sólidos voláteis, consequentemente é obtido pela diferença entre os valores do teor de sólidos toais e teor de sólidos fixos. Assim, o teor de sólidos voláteis, foi obtido pela seguinte expressão:

$$
\% \text { resíduo volátil }=\frac{(m 2-m 3) \times 100}{m 2}
$$




\section{Potencial hidrogeniônico $(\mathrm{pH})$}

O pH foi medido, conforme instruções apresentadas na Norma NBR 7353/2014 (Soluções aquosas Determinação do pH com eletrodos de vidro), mas considerando o Manual de Métodos Analíticos Oficiais para Fertilizantes e Corretivos do Ministério da Agricultura, Pecuária e Abastecimento (MAPA). Neste contexto, considerando o composto como fertilizante orgânico, as leituras de $\mathrm{pH}$, foram realizadas suspendendo as amostras em solução de Cloreto de Cálcio $\left(\mathrm{CaCl}_{2}\right)$ na concentração de 0,01 mol..-1. Para tanto, pesou-se 10g ( $\pm 1 \mathrm{mg}$ ) de cada amostra 'in natura', e transferiu-se para béqueres de $100 \mathrm{~mL}$. Logo após, foi adicionado $50 \mathrm{~mL}$ de solução de $\mathrm{CaCl}_{2}$ 0,01 mol.L-1 e efetuou-se a homogeneização. Após a homogeneização, esta solução de amostra e $\mathrm{CaCl}_{2}$, ficou descansando por 30 minutos, onde de 10 em 10 minutos efetuou-se a agitação deste material. Em seguida, foram feitas as leituras dos pH, expressando o resultado com a indicação de ' $\mathrm{pH}$ em solução de $\mathrm{CaCl}_{2}$ a 0,01 mol. $\mathrm{L}^{-1}$ '.

\section{Condutividade elétrica (C.E.)}

A condutividade elétrica, foi medida, conforme instruções apresentadas na Norma NBR 14340/1999, Água - Determinação da condutividade e da resistividade elétrica, mas considerando o Manual de Métodos Analíticos Oficiais para Fertilizantes e Corretivos do Ministério da Agricultura, Pecuária e Abastecimento (MAPA).

A condutividade elétrica, foi medida em condutivímetro digital com célula de condutividade, considerando o Manual de Métodos Analíticos Oficiais para Fertilizantes e Corretivos do Ministério da Agricultura, Pecuária e Abastecimento (MAPA).

Para tal, pesou-se $10 \mathrm{~g}$ de cada amostra, com precisão de 0,1 mg, e transferiu-se para béqueres de $200 \mathrm{~mL}$. Logo após foi adicionado $100 \mathrm{~mL}$ de água deionizada e efetuou-se a homogeneização. Após a homogeneização, esta solução de amostra e água deionizada ficou em repouso por 10 minutos. Em seguida, procedeu-se com as leituras de condutividade elétrica.

\section{RESULTADOS E DISCUSSÃO}

A avaliação da eficiência do biorremediador comercial 'Acelerador de compostagem' da Embiotic ${ }^{\circledR}$ Line no processo de bioxidação de lodo de esgoto sanitário, teve como parametro a avaliação da temperatura e as análises de umidade, pH, C.E. e teores de sólidos (totais, fixos e voláteis) efetuadas durante o processo de compostagem, bem como a avaliação das análises efetuadas no fertilizante orgânico obtido na finalização do processo de compostagem.

Cabe ressaltar que as leiras foram compostas pelos mesmos resíduos (lodo de esgoto e casca de eucalipto) e todos os processos envolvidos ocorreram igualmente e simultaneamente no mesmo período. Em relação a avaliação do monitoramento das temperaturas, ocorreram oscilações conforme exposto no Gráfico 1. 


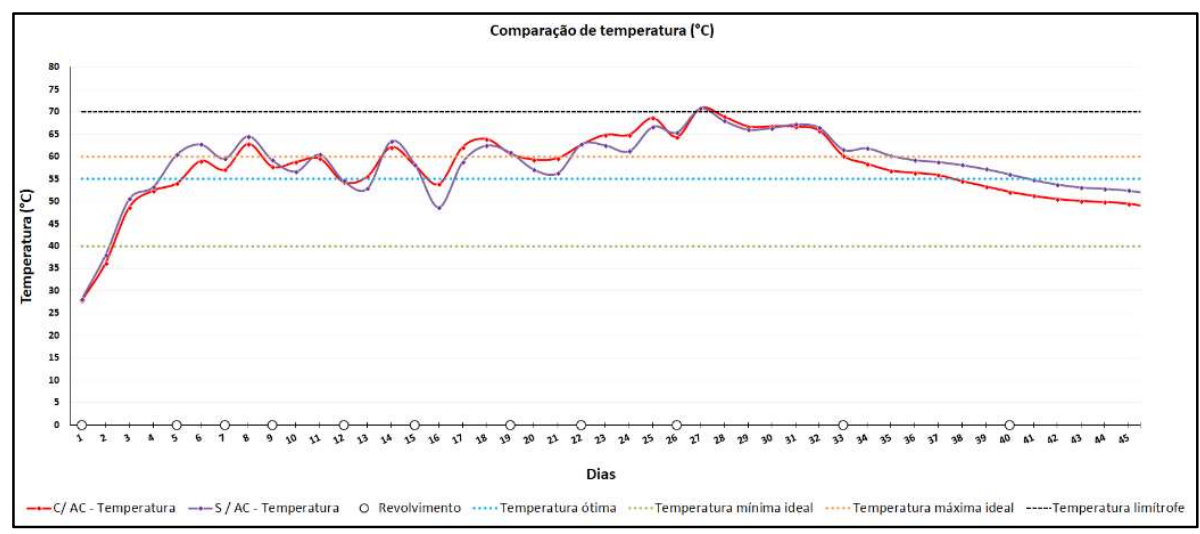

Gráfico 1: Comparativo das oscilações de temperatura nas leiras de compostagem.

Como pode ser visto no gráfico de comparação de temperatura, a leira que recebeu o biorremediador, representado pela curva $(C / A C)$ e da leira que não recebeu o biorremediador, representado pela curva $(\mathrm{S} / \mathrm{AC})$, tiveram suas temperaturas como esperado, iniciando na fase mesófilica. Entretanto, evidencia-se que as temperaturas ultrapassam a temperatura mínima ideal de $40 \circ \mathrm{C}$ em apenas 48 horas, adentrando na fase termófilica em ambas as leiras.

Entretanto, no início do processo de compostagem, evidencia-se uma maior temperatura, porém não significativamente expressiva na curva (S/AC) e este comportamento segue assim até o nono dia do processo de degradação, onde as temperaturas das curvas (S/AC) e (C/AC) se igualam. Verifica-se uma oscilação (diminuição e aumento) de temperaturas nas leiras, como esperado, decorrente do processo de revolvimento. A cada revolvimento, constatou-se uma depleção de temperatura com rápida retomada, onde a curva $(\mathrm{S} / \mathrm{AC})$ apresentou menores variações.

Contudo, o fato contribuinte para a curva (C/AC) apresentar maiores oscilações, deveu-se a inoculação de biorremediador antes de cada revolvimento, o que causou um resfriamento ligeiramente maior, porem momentâneo na leira. Além disso, a partir do nono dia do processo, as temperaturas da curva $(S / A C)$, apresentaram uma pequena vantagem em relação as temperaturas da curva (C/AC), onde a leira sem inoculação alcançou temperaturas acima de 65 C com uma vantagem de 48 horas em relação a leira inoculada.

No entanto, a partir do momento que a curva $(C / A C)$, alcança a temperatura de $65^{\circ} \mathrm{C}$, ambas as curvas permanecem por cerca de 8 dias na faixa de temperaturas compreendidas entre 65 e $70 \circ$ C, caracterizando o processo de higienização do composto, ou seja, momento em que a bioestabilização ocorre. Logo a após, começa a fase de resfriamento (segunda fase mesófilica), quando a atividade dos microrganismos termofílicos cessa devido ao esgotamento dos substratos, dando início ao processo de maturação do composto.

Assim, em relação a comparação das temperaturas nas leiras (inoculada e controle), os resultados não apresentaram significativas mudanças nas faixas de temperaturas quando confrontadas, assim não foi constatado contribuições que justifiquem o emprego da inoculação do biorremediador. Em relação ao teor de umidade, o Gráfico 2 apresenta as variações obtidas. 


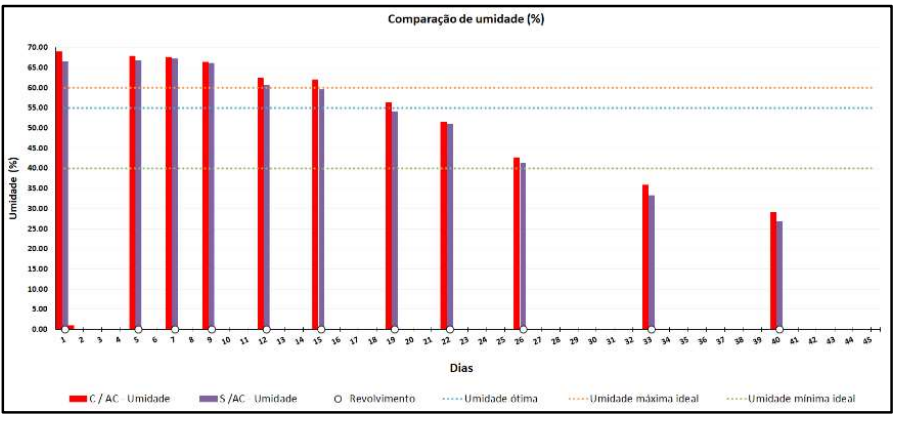

Gráfico 2: Variação do teor de umidade na leira de compostagem do lodo de esgoto sanitário.

Em relação aos teores de umidade, do início ao fim do processo, não foi evidenciado mudanças significativas em ambas as leiras (inoculada e controle), como era de se esperar, após avaliação da oscilação de temperatura. Entretanto, ficou bem evidente durante todo o processo de compostagem, o fato da leira que recebeu o biorremediador apresentar teores de umidade ligeiramente maior que a leira que não recebeu o biorremediador.

Entretanto, ambas as leiras, durante todo o processo de compostagem, permaneceram dentro da faixa ideal de umidade (40 a 60\%), visto que há perdas de água no processo devido a evaporação ocasionada pelo material em decomposição. Assim, em relação a comparação dos teores de umidade nas leiras (inoculada e controle), os resultados não apresentaram significativas mudanças nos teores de umidade quando confrontadas, assim não foi constatado contribuições que justifiquem o emprego da inoculação do biorremediador. Em relação ao potencial hidrogeniônico $(\mathrm{pH})$ e a condutividade elétrica (C.E.), as variações são apresentas no Gráfico 3 e 4, respectivamente.

Por meio do gráfico do $\mathrm{pH}$, verifica-se que o potencial hidrogeniônico no início do processo apresenta valores mais altos, e conforme o tempo de compostagem avança, esses valores diminuem. Essa diminuição nos valores de pH é característica do processo fermentativo. Assim em contrapartida, os valores da C.E. começam baixos e conforme o tempo de compostagem avança, esses valores aumentam devido ao processo fermentativo que libera íons.

Deste modo o pH e a C.E. são diretamente proporcionais e apresentaram resultados satisfatórios, entretanto, avaliando-se isoladamente o pH e a C.E., temos como resultado, que o uso do biorremediador não apresentou mudanças significativas quando confrontados em relação as leiras (inoculada e controle) que justifiquem seu uso.

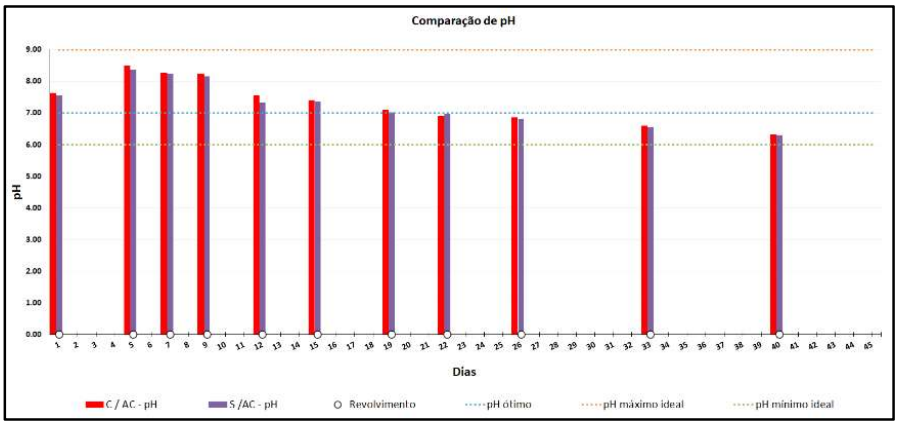

Gráfico 3: Variação do pH na leira de compostagem do lodo de esgoto sanitário. 


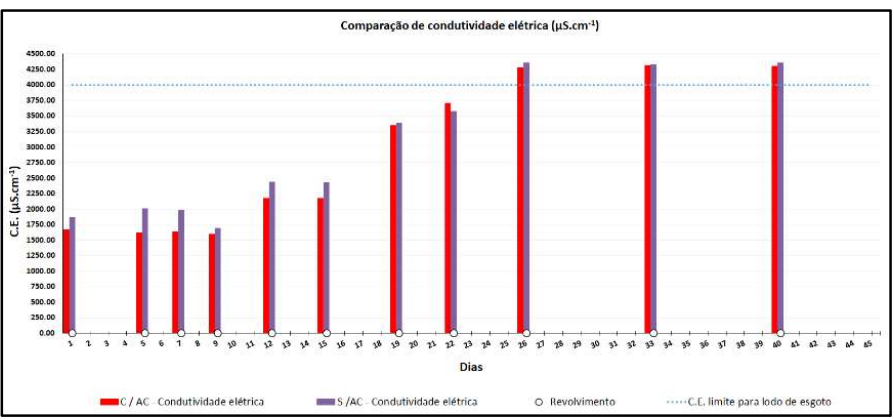

Gráfico 4: Variação condutividade elétrica na leira de compostagem do lodo de esgoto sanitário.

Por meio do gráfico do $\mathrm{pH}$, verifica-se que o potencial hidrogeniônico no início do processo apresenta valores mais altos, e conforme o tempo de compostagem avança, esses valores diminuem. Essa diminuição nos valores de pH é característica do processo fermentativo. Assim em contrapartida, os valores da C.E. começam baixos e conforme o tempo de compostagem avança, esses valores aumentam devido ao processo fermentativo que libera íons.

Deste modo o pH e a C.E. são diretamente proporcionais e apresentaram resultados satisfatórios, entretanto, avaliando-se isoladamente o pH e a C.E., temos como resultado, que o uso do biorremediador não apresentou mudanças significativas quando confrontados em relação as leiras (inoculada e controle) que justifiquem seu uso.

Estas observações também foram feitas no estudo feito por Nord (2013), ao usar o mesmo biorremediador, e de acordo com o autor, o uso do biorremediador não apresentou melhoras significativas em relação a pH e consequentemente a C.E. Em relação aos teores de sólidos totais (ST), fixos (SF) e voláteis (SV), são apresentados nos Gráficos (5, 6 e 7) respectivamente os valores obtidos. Conforme apresentado nos gráficos de ST, SF e SV, os teores dos mesmos não apresentaram uma mudança significativa quando confrontados em relação as leiras (inoculada e controle) que justifique o uso do biorremediador.

Considerando as avaliações apresentadas em relação ao comportamento da temperatura, umidade, pH, C.E., ST, SF e SV, o uso do biorremediador apresentou-se irrelevante para o sistema, uma vez que os próprios microrganismos presentes no processo são suficientes para os processos de degradação da matéria orgânica. Assim, como Kiehl (2012), em seus estudos, aponta que pesquisas realizadas em diversos países já demostraram a inoperância desses produtos quando inoculados em diversos resíduos.

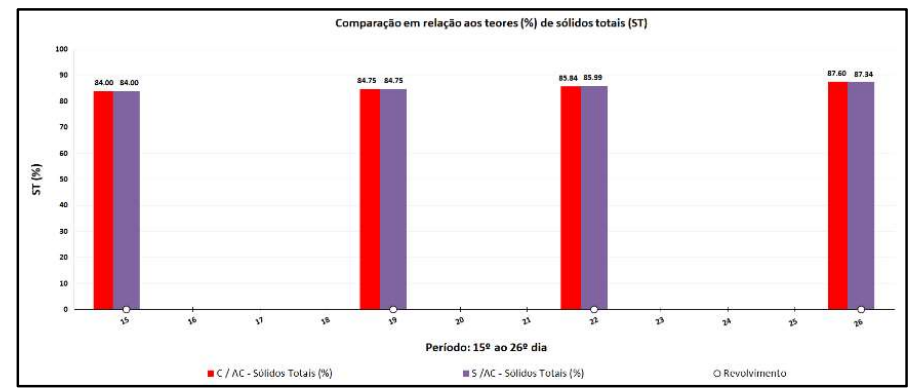

Gráfico 5: Teores de sólidos totais na leira de compostagem do lodo de esgoto sanitário. 


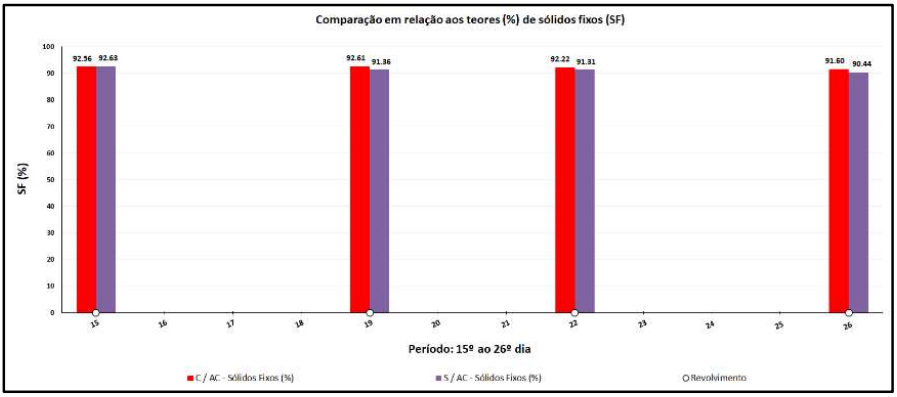

Gráfico 6: Teores de sólidos fixos na leira de compostagem do lodo de esgoto sanitário.

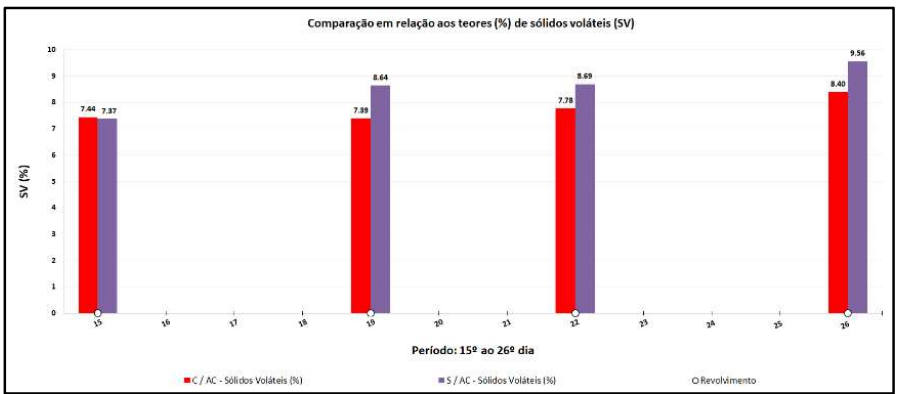

Gráfico 7: Teores de sólidos voláteis na leira de compostagem do lodo de esgoto sanitário.

\section{CONCLUSÕES}

Conclui-se neste projeto que a inoculação do biorremediador 'Acelerador de Compostagem' da Embiotic ${ }^{\circledR}$-Line, foi irrelevante, considerando as circunstâncias em que foi desenvolvido o processo de compostagem com lodo de esgoto sanitário. Neste sentido, apesar do biorremediador ser constituído por microrganismos efetivos (EM), o mesmo não propiciou a compostagem acelerada, todavia, cabe salientar que não corroborou em momento algum no sentido de dificultar o processo de compostagem.

\section{REFERÊNCIAS}

ANDRADE, J. A.; AUGUSTO, F.; JARDIM, I. C. S. F.. Biorremediação de solos contaminados por petróleo e seus derivados. Eclética Química, Araraquara, v.35, n.3, p.17-43, 2010. DOI: http://dx.doi.org/10.1590/S010046702010000300002

ABNT. Associação Brasileira de Normas Técnicas. NBR 10664:1989 - Águas - Determinação de resíduos (sólidos) Método gravimétrico. Rio de Janeiro: ABNT, 1989. 7p.

ABNT. Associação Brasileira de Normas Técnicas. NBR 14340:1999 - Água - Determinação da condutividade e da resistividade elétrica. Rio de Janeiro: ABNT, 1999. 3p

ABNT. Associação Brasileira de Normas Técnicas. NBR 7353:2014 - Soluções aquosas - Determinação do pH com eletrodos de vidro. Rio de Janeiro: ABNT, 2014. 8p.

BUDZIALK, C. R.; MAIA, C. M. B. F.; MANGRICH, A. S.. Transformações químicas da matéria orgânica durante a compostagem de resíduos da indústria curtumeira. Química Nova, São Paulo, v.27, n.3, p.399-403, 2004. DOI: http://dx.doi.org/10.1590/S0100-40422004000300007

CARVALHO, P. C. T.. Compostagem. In: TSUTIYA, M. T.; Comparini, J. B.; Sobrinho, P. A. et al. Biossólidos na agricultura. 2 ed. São Paulo: ABES, 2002.
FELS L. E.; ZAMAMA, M.; ASLI, A. E.; HAFIDI, M.. Assessment of biotransformation of organic matter during COcomposting of sewage sludge-lignocelullosic waste by chemical, FTIR analyses, and phytotoxity tests. International Biodeterioration \& Biodegradation, v. 7, p.128-137, 2014 DOI: https://doi.org/10.1016/j.ibiod.2013.09.024

FERNANDES, F.; SILVA, S. M. C. P.. Manual prático para compostagem de biossólidos. Rio de Janeiro: ABES, 1999.

INSAM, H.; BERTOLDI, M.. Microbiology of the composting process. Waste Management Series: Compost Science and Technology, v.8, p.25-48, 2007. DOI: https://doi.org/10.1016/S1478-7482(07)80006-6

KIEHL, E. J.. Manual de Compostagem: Maturação e Qualidade do Composto. Piracicaba: Gráfica e Editora Degaspari, 2012.

PAZ, V.; UMBIDES, R.; MISHIMA, H.; SÁNCHEZ, A. P.; PINTO. M. I. S.. Tratamiento de los residuos urbanos en Santiago del Estero (Argentina): Una experiencia piloto. Revista Técnica, La Rioja, n.71, p.40-47, 2003.

QIAN, X.; SHEN, G.; WANG, Z.; GUO, C.; LIU, Y.; LEI, Z.; ZHANG, Z.. Co-composting of livestock manure with rice 
straw: Characterization and establishment of maturity evaluation system. Waste Management, v.34, n.2, p.530-
535, 2014. DOI:

https://doi.org/10.1016/i.wasman.2013.10.007

A CBPC - Companhia Brasileira de Produção Científica (CNPJ: 11.221.422/0001-03) detém os direitos materiais desta publicação. Os direitos referem-se à publicação do trabalho em qualquer parte do mundo, incluindo os direitos às renovações, expansões e disseminações da contribuição, bem como outros direitos subsidiários. Todos os trabalhos publicados eletronicamente poderão posteriormente ser publicados em coletâneas impressas sob coordenação da Sustenere Publishing, da Companhia Brasileira de Produção Científica e seus parceiros autorizados. Os (as) autores (as) preservam os direitos autorais, mas não têm permissão para a publicação da contribuição em outro meio, impresso ou digital, em português ou em tradução. 